\title{
Erratum zu: Über Ähnlichkeiten heutiger Coachingverständnisse zum Supervisionsdiskurs zwischen 1945 und 1975. Ein ideengeschichtlicher Essay
}

\author{
Volker Jörn Walpuski ${ }^{1}$ (D) \\ Online publiziert: 31 . Juli 2021 \\ (c) Der/die Autor(en) 2021
}

\section{Erratum zu:}

\section{Coaching Theor. Prax. 2021}

https://doi.org/10.1365/s40896-021-00053-5

In der Originalversion des Beitrags waren leider einige Fehler in der Literatur. Sie sollte wie im Literaturverzeichnis dargestellt aussehen.

Der Originalbeitrag wurde korrigiert.

Open Access Dieser Artikel wird unter der Creative Commons Namensnennung 4.0 International Lizenz veröffentlicht, welche die Nutzung, Vervielfältigung, Bearbeitung, Verbreitung und Wiedergabe in jeglichem Medium und Format erlaubt, sofern Sie den/die ursprünglichen Autor(en) und die Quelle ordnungsgemäß nennen, einen Link zur Creative Commons Lizenz beifügen und angeben, ob Änderungen vorgenommen wurden.

Die in diesem Artikel enthaltenen Bilder und sonstiges Drittmaterial unterliegen ebenfalls der genannten Creative Commons Lizenz, sofern sich aus der Abbildungslegende nichts anderes ergibt. Sofern das betreffende Material nicht unter der genannten Creative Commons Lizenz steht und die betreffende Handlung nicht nach gesetzlichen Vorschriften erlaubt ist, ist für die oben aufgeführten Weiterverwendungen des Materials die Einwilligung des jeweiligen Rechteinhabers einzuholen.

Weitere Details zur Lizenz entnehmen Sie bitte der Lizenzinformation auf http://creativecommons.org/licenses/by/4.0/deed.de.

Die Online-Version des Originalartikels ist unter https://doi.org/ 10.1365/s40896-021-00053-5 zu finden.

Volker Jörn Walpuski

volker.walpuski@hs-hannover.de

1 Fakultät V Diakonie, Gesundheit und Soziales, Hochschule Hannover, Blumhardtstraße 2, 30625 Hannover, Deutschland

\section{Literatur}

\section{Verwendete Literatur}

Andriessen, H. (1978). Pastorale Supervision: Praxisberatung in der Kirche. München, Mainz: Kaiser.

Arbeitskreis Soziale Fortbildung Bremen (Hrsg.). (1953). Verstehen um zu Helfen: Ein Beitrag zur Arbeit mit Menschen hervorgegangen aus dem Bremer Seminar 1953. Bremen-Achim: Buchdruckerei Joh. Oltmanns.

Austermann, F. (2013). Coaching, das Personal entwickelt, Personen ausschließt und Personenentwicklung instrumentalisiert. Ein diskursanalytischer Blick auf Schreyöggs Beratungsverständnis und ihren Rückgriff auf Neubergers „Personalentwicklung“. FoRuM Supervision - Zeitschrift für Beratungswissenschaft und Supervision, 21(42), 35-49. https://doi.org/10.4119/fs-2208.

Baltussen, C.W.M. (1953). Summer report, 1953 to the United Nations. Nijmegen: Katholiek Dokumentatie Centrum.

Bang, R. (1967). Autorität, Gewissensbildung, Toleranz, drei Grundprobleme der Einzelfallhilfe: Ein Beitrag zum Selbstverständnis der Helferpersönlichkeit. Einzelfallhilfe, Casework, in der Praxis der Sozialarbeit. Bd. 3. München, Basel: Ernst Reinhardt.

Belardi, N. (1992). Supervision: Von der Praxisberatung zur Organisationsentwicklung. Paderborn: Junfermann.

v. Bethusy-Huc, V. G. (1971). Buchrezension: Brief and Extended Casework by W. J. Reid, Ann W. Shyne. ARSP: Archiv für Rechtsund Sozialphilosophie, 57(3), 455-456.

Böning, U., \& Kegel, C. (2013). Psychometrische Persönlichkeitsdiagnostik. In H. Möller \& S. Kotte (Hrsg.), Diagnostik im Coaching (S. 81-99). Berlin Heidelberg: Springer. https://doi.org/10.1007/ 978-3-642-37966-6_6.

Bourdieu, P. (1993). Narzissitische Reflexivität und wissenschaftliche Reflexivität. In E. Berg \& M. Fuchs (Hg.), Kultur, soziale Praxis, Text: Die Krise der ethnographischen Repräsentation (S. 365-374). Frankfurt am Main: Suhrkamp.

Braunthal, H. (1952). A casework training course as a group-therapeutic experience. International Journal of Group Psychotherapy, 2(3), 239-244. https://doi.org/10.1080/00207284.1952. 11508445

Bröckling, U. (2017). Gute Hirten führen sanft: Über Menschenregierungskünste. Berlin: Suhrkamp.

Bundesagentur für Arbeit (2021). Geschäftsbericht der Bundesagentur für Arbeit 2020. Nürnberg. https://www.arbeitsagentur.de/ datei/geschaeftsbericht-2020_ba146981.pdf. Zugegriffen: 23. Juni 2021. 
Burkart, G. (2006). Einleitung. In G. Burkart (Hrsg.), Die Ausweitung der Bekenntniskultur - neue Formen der Selbstthematisierung? (S. 7-40). Wiesbaden: VS.

v. Caemmerer, D. (1952). Probation. Aufbau und Praxis der englischen Systems der Bewährungshilfe. München, Düsseldorf: Steinebach.

v. Caemmerer, D. (1966). Akademiekurs über Praxisanleitung (Supervision) vom 1. November 1964-15. Mai 1966. Nachrichtendienst des Deutschen Vereins Für Öffentliche und Private Fürsorge (ndv), 46(9), 273-276.

v. Caemmerer, D. (Hrsg.). (1970). Praxisberatung (Supervision): Ein Quellenband. Lambertus.

v. Caemmerer, D., \& Schiller, H. (1953). Aufbaulehrgänge für Berliner Sozialarbeiter. Soziale Arbeit, 2(6), 252-263.

Dreier, W., \& Steinkamp, H. (1969). Wirkmacht des Sozialen. Mit einem Erfahrungsbericht über die Fortbildung in sozialer Gruppenarbeit. Schriftenreihe der Akademie für Jugendfragen, Bd. 3. Münster: Regensberg.

Duensing, F. (1920). Ziele und Aufgaben der Sozialen Frauenschule. Rede von Dr. jur. Frieda Duensing. In Soziale Frauenschule der Stadt München (Hrsg.), Programm, nebst einem Anhang, enthaltend die Eröffnungsreden von Dr. Georg Kerschensteiner und Dr. jur. Frieda Duensing (S. 14-24). München: Carl Gerber.

Etzioni, A. (1964). Modern organizations. Foundations of modern sociology series. Englewood Cliffs: Prentice-Hall.

Föllmer, M. (1977). Supervision in der amerikanischen Sozialarbeit. Neue Praxis, 7(1), 4-17.

Freier, C. (2016). Soziale Aktivierung von Arbeitslosen? Praktiken und Deutungen eines neuen Arbeitsmarktinstruments. Gesellschaft der Unterschiede, Bd. 38. Bielefeld: transcript.

Geißler, H. (2017). Die Grammatik des Coachens: Eine empirische Rekonstruktion. Wiesbaden: Springer. https://doi.org/10.1007/9783-658-09640-3

Graf, E.-M., \& Fleischhacker, M. (2020). „Wenn ich es nicht schaffe, liegt es an meiner Person und nicht an meiner Leistung" - Die Individualisierung struktureller Probleme im Coaching weiblicher Führungskräfte. Genderlinguistische und gendertheoretische Erkenntnisse für die Praxis. Coaching | Theorie \& Praxis, 6(1), 111-133. https://doi.org/10.1365/s40896-020-00034-0.

Grant, A. (2018). Zielperspektiven in die Coaching-Praxis integrieren - Ein integratives Modell zielorientierten Coachings. Coaching | Theorie \& Praxis, 4(1), 1-15. https://doi.org/10.1365/s40896017-0020-X.

Greif, S., \& Benning-Rohnke, E. (2015). Konsequente Umsetzung von Zielen durch Coaching. Coaching | Theorie \& Praxis, 1(1), 25-35. https://doi.org/10.1365/s40896-015-0003-8.

Griewatz, H.-P. (2018). „Supervision ist Coaching für helfende Berufe", oder: Über die Invasion gouvernementaler Beratungspraxis Eine Polemik. FoRuM Supervision - Onlinezeitschrift für Beratungswissenschaft und Supervision, 26(51), 75-81.

Griewatz, H.-P., \& Walpuski, V.J. (2017). „Foucault im Jobcenter“. Supervision in einem widersprüchlichen gesellschaftlichen Feld (Teil 1). Forum Supervision. Onlinezeitschrift Für Beratungswissenschaft und Supervision, 25(50), 12-30. https://doi.org/10. 4119/fs- 2337.

Griewatz, H.-P., \& Walpuski, V.J. (2018). „Foucault im Jobcenter“. Supervision in einem widersprüchlichen gesellschaftlichen Feld (Teil 2). Forum Supervision. Onlinezeitschrift Für Beratungswissenschaft und Supervision, 26(51), 39-74. https://doi.org/10. 4119/fs-2331.

Gröning, K. (2013). Supervision: Traditionslinien und Praxis einer reflexiven Institution. Therapie \& Beratung. Gießen: Psychosozial.

Gröning, K. (2015). Entwicklungslinien pädagogischer Beratung. Zur Geschichte der Erziehungs-, Berufs- und Sexualberatung in Deutschland. Erweiterte und korrigierte Neuauflage. Gießen: Psychosozial-Verlag (Therapie \& Beratung).

Gröning, K. (2016). Sozialwissenschaftlich fundierte Beratung in Pädagogik, Supervision und Sozialer Arbeit. Gießen: PsychosozialVerlag (Therapie \& Beratung).
Gross, P.-P., \& Stephan, M. (2012). Die Entwicklung des deutschen Coaching-Marktes und das Marktpotential von Coaching mit neuen Medien - eine ökonomische Analyse. In H. Geißler \& M. Metz (Hrsg.), E-Coaching und Online-Beratung: Formate, Konzepte, Diskussionen (S. 319-338). Wiesbaden: Springer VS.

Hamilton, G. (1950). Die helfende Beziehung. Helping People-The Growth of a Profession, in: Journal of Social Casework, October 1948, p. 291-299. In H. Kraus (Hrsg.), Casework in USA: Theorie und Praxis der Einzelhilfe. Wissenschaftliche Schriftenreihe des Instituts zur Förderung öffentlicher Angelegenheiten. (S. 47-55). Frankfurt am Main: Metzner.

Heekerens, H.-P. (2016). Psychotherapie und Soziale Arbeit: Studien zu einer wechselvollen Beziehungsgeschichte. Schriften zur psychosozialen Gesundheit. Weitramsdorf-Weidach: ZKS. http:// www.publish-Books.de/zksverlag/books/ID48552. Zugegriffen: 23. Dez. 2020.

James, M. (1954). Eind-rapport: Observatie verricht van 5 Januari 1953 tot 4 Februari 1954 in Amerika in het kader van het "United Nations Social Welfare Fellowship Programme" over het onderwerp "Casework Methods and Methods of Supervision" (Serie A No. 28). 's-Gravenhage: Ministerie van Maatschappelijk Werk. Afdeling Maatschappelijk Opbouwwerk.

Judd, J., Kohn, R.E., \& Schulman, G. L. (1962). Group supervision: a vehicle for professional development. Social Work, 7(1), 96-102.

Judd, J., Kohn, R.E., \& Schulman, G.L. (1970). Praxisberatung in Gruppen (Group Supervision): ein Mittel zur beruflichen Entwicklung. In D. v. Caemmerer (Hrsg.), Praxisberatung (Supervision): Ein Quellenband (S. 230-243). Freiburg im Breisgau: Lambertus.

Junk, M. (1955). Jahrestagung der Konferenz der Deutschen Wohlfahrtsschulen in Berlin vom 27.09. bis 01.10.1954. Blätter der Wohlfahrtspflege, 102(3), 87-89.

Kadushin, A., \& Harkness, D. (2014). Supervision in social work (5. Aufl.). New York: Columbia University Press.

Kappeler, M. (2001). Selbstaufklärung statt Identitätspolitik - Supervision und Geschichtsschreibung als Möglichkeiten der Selbst-Reflexion in der Sozialen Arbeit. FoRuM Supervision - Zeitschrift für Beratungswissenschaft und Supervision, 9(18), 9-19.

Kasius, C. (1951). A comparison of diagnostic and functional casework concepts: report of the Familiy service association of America committee to study basic concepts in casework practice. New York: Family Service Association of America.

Kersting, H.J. (1975). Kommunikationssystem Gruppensupervision: Aspekte eines Lernlehrverfahrens. Freiburg im Breisgau: Lambertus.

Koch, P., \& Oesterreicher, W. (1985). Sprache der Nähe - Sprache der Distanz. Mündlichkeit und Schriftlichkeit im Spannungsfeld von Sprachtheorie und Sprachgeschichte. Romanistisches Jahrbuch, $36,15-43$.

Kramann, H. (2020). Do it yourself. Selbstsupervision und Selbstcoaching: Ein Methodenkoffer für mehr Souveränität und Leichtigkeit im Beruf. Göttingen: Vandenhoeck \& Ruprecht.

Krämer, K. (1983). Von der Ungeduld, warten zu können. Supervision, 2(3), 43-60.

Krämer, R. (1982). Teamsupervision innerhalb eines Großunternehmens. Supervision, 1(2), 17-29.

Krause, M. (1964). Ausbildung von Führungskräften in der Sozialarbeit. Europäisches Seminar der Vereinten Nationen vom 22.-31.10.63 in Amersfoort, Holland. Soziale Arbeit, 13(2), $53-60$.

Kutter, P., Schäfer, A., \& Roth, J. K. (1977). Erfahrungen und Erfolge mit psychoanalytisch orientierter Supervision nebenberuflicher Studienberater an der Universität Frankfurt/Main. Kooperationssystem Studienberatung

Lander, H. (1977). Demokratisierung durch Partizipationshandeln mit Hilfe des Lerninstrumentes Supervision: Dargestellt am Praxisfeld Elementar-Erziehung, insbesondere Kindergartenbeirat [In- 
auguraldissertation]. Frankfurt am Main: Johann Wolfgang Goethe-Universität.

Leuschner, G. (1970). Gruppendynamik im Laboratorium. Sozial. Berufsverband Katholischer Sozialarbeiterinnen und Sozialarbeiter, 22(5), 81-89.

Leuschner, G. (1999). Akquisition und Kontrakt im Gegenwind des Zeitgeistes. FoRuM Supervision - Zeitschrift für Beratungswissenschaft und Supervision, 7(14), 5-23.

Link, J. (2009). Versuch über den Normalismus: Wie Normalität produziert wird (3. Aufl.). Vandenhoeck \& Ruprecht.

Loebbert, M. (2016). Wie Supervision gelingt: Supervision als Coaching für helfende Berufe. essentials. Berlin Heidelberg: Springer. https://doi.org/10.1007/978-3-658-13106-7.

Lohl, J. (2019). „,.. und ging ins pralle Leben “: Facetten einer Sozialgeschichte der Supervision. Interdisziplinäre Beratungsforschung, Bd. 15. Göttingen: Vandenhoeck \& Ruprecht.

Lowy, L. (1977). Supervision: Ein agogischer Lehr- und Lernprozeß. In D. v. Caemmerer, F. W. Kröger, H. Lander, H. Schiller, H. Merkelbach \& U. Walz (Hrsg.), Supervision, ein berufsbezogener Lernprozeß (S. 8-18). Wiesbaden-Dotzheim: Haus Schwalbach.

Luhmann, N. (1971). Moderne Systemtheorie als Form gesamtgesellschaftlicher Analyse. In J. Habermas \& N. Luhmann (Hrsg.), Theorie der Gesellschaft oder Sozialtechnologie. Was leistet die Systemforschung? (S. 7-24). Frankfurt am Main: Suhrkamp.

Maraun, E. (1952). Casework und Supervision in der amerikanischen Jugendfürsorge. Praxis der Kinderpsychologie und Kinderpsychiatrie, 1, 243-246.

Melzer, G. (1964). Die Ausbildung von leitendem Personal für soziale Einrichtungen: Europäisches Seminar der Vereinten Nationen vom 21.-31. Oktober 1964 in Amersfoort/Niederlande. Nachrichtendienst des Deutschen Vereins Für Öffentliche und Private Fürsorge (ndv), 44(2), 78-81.

Melzer, G. (1981a). Beratung als Leitungsaufgabe. Die Entwicklung der Praxisberaterkurse der Akademie des Deutschen Vereins von 1964 bis 1980. Nachrichtendienst des Deutschen Vereins Für Öffentliche und Private Fürsorge (ndv), 61(1), 20-26.

Melzer, G. (1981b). Leitung und Führung durch Beratung. Sozialpädagogische Blätter, 6(3), 75-80.

Moede, W. (1930). Lehrbuch der Psychotechnik. Berlin: Springer.

Möller, H., \& Zimmermann, J. (2020). Schwierige Situationen im Business-Coaching: Praxisbeispiele, Perspektiven und Handlungsmöglichkeiten. Berlin: Springer.

Möller, H. (2011). Astrid Schreyögg. Eine der großen Damen der Supervision wird 65 Jahre alt! Journal Supervision, (3), 19.

Morczinek, H. (1956). Gruppenarbeit unter Praxisberatung. Die Soziale Arbeit, 14, 15-18.

Neuberger, O. (1990). Der Mensch ist Mittelpunkt. Der Mensch ist Mittel. Punkt. Acht Thesen zum Personalwesen. Personalführung, 23(1), 3-10.

Neuberger, O. (1994 [1991]). Basistexte Personalwesen (2. Aufl.). Personalentwicklung, Bd. 2. Stuttgart: F. Enke.

Neuffer, M. (1990). Die Kunst des Helfens: Geschichte der sozialen Einzelhilfe in Deutschland. Edition Sozial. Weinheim: Beltz.

o. V. (1929). Rundschau. Deutsche Zeitschrift für Wohlfahrtspflege, 5(5), 302. https://www.dzi.de/wp-content/dzw/Jg05/05/ Wohlfahrtspflege_Jg05_05.pdf. Zugegriffen: 11. Feb. 2021.

Reid, W. J., \& Shyne, A.W. (1969). Brief and extended casework. New York, London: Colombia University Press.

Richmond, M.E. (1917). Social diagnosis. SAGE.

Ringshausen-Krüger, M. (1977). Die Supervision in der deutschen Sozialarbeit: Entwicklung von Konzeptionen, Methoden und Strukturen 1954-1974; eine textanalytische Untersuchung. Dissertation. Frankfurt am Main: Universität Frankfurt am Main, Fachbereich 04 - Erziehungswissenschaften.

Roesinger, A. (1955). Die Einrichtung der Supervision in Mannheim und die Erfahrungen hieraus. Katholische Sozialarbeit, 7(2), $25-27$.
Roesinger, A. (1957). Über die Weiterführung der Supervision in Mannheim. Katholische Sozialarbeit, 9(4), 69-72.

Sauter, W. (1994). Vom Vorgesetzten zum Coach der Mitarbeiter: Handlungsorientierte Entwicklung von Führungskräften. Weinheim: Dt. Studien-Verlag.

Scherpner, H. (1927). Formen persönlicher Fürsorge in den Vereinigten Staaten (social case work). Berlin: Vahlen. Sonderdruck aus Freie Wohlfahrtspflege (Band 1 und 2)

Scherz, F.H. (1958). A concept of supervision based on definitions of job responsibility. Social Casework, 39(8), 435-443.

Scherz, F.H. (1970). Vorschläge zur Praxisberatung - auf der Grundlage neu definierter Verantwortungsbereiche. In D. v. Caemmerer (Hrsg.), Praxisberatung (Supervision): Ein Quellenband (S. 195-212). Freiburg im Breisgau: Lambertus.

Schiller, H. (1988). Dr. Dora von Caemmerer und ihr Beitrag zur Geschichte der deutschen Sozialausbildung und zur Einführung von Supervision. Supervision, 6(14), 65-71.

Schreyögg, A. (2009). Besonderheiten des Coaching - Unterschiede zur Supervision. In H. Pühl (Hrsg.), Handbuch Supervision und Organisationsentwicklung. 3. aktualisierte und erweiterte Auflage (S. 196-208). Berlin: Leutner.

Schreyögg, A. (2013). Coaching und/oder Supervision. Organisationsberatung, Supervision, Coaching, 20(2), 231-237. https://doi.org/ 10.1007/s11613-013-0326-0

Schultze, A. (1959). Social Work Education in the United States of America and in the Federal Republic of Germany in the Light of International Social Work Education 1959 [unveröffentlichte Masterarbeit]. Salt Lake City: University of Utah.

Schultze, A. (1971). Wandlungen in der amerikanischen Sozialarbeit. Soziale Arbeit, 20(4), 152-159.

de Shazer, S. (1975). Brief therapy: two's company. Family Process, 14(1), 79-93. https://doi.org/10.1111/j.1545-5300.1975.00079.x.

de Shazer, S. (1978). Brief therapy with couples. International Journal of Family Counseling, 6(1), 17-30. https://doi.org/10.1080/ 01926187808250271.

Steinhardt, K. (2007). Psychoanalytisch orientierte Supervision - auf dem Weg zu einer Profession? Zur historischen, professionstheoretischen und empirischen Fundierung von psychoanalytisch orientierter Supervision. Zugleich Dissertation Universität Wien (2. Aufl.). Psychoanalytische Pädagogik, Bd. 19. Wien: Psychosozial-Verlag.

Steinkamp, H. (1973). Gruppendynamik und Demokratisierung: Ideologiekritische und sozialethische Studien zur empirischen und angewandten Kleingruppenforschung. Gesellschaft und Theologie Abteilung Sozialwissenschaftliche Analysen, Bd. 10. München, Mainz: Kaiser, Matthias-Grünewald-Verl.

Taft, J. (1933). The dynamics of therapy in a controlled relationship. New York: Macmillan.

Towle, C. (1967). The learner in education for the professions as seen in education for social work (6. Aufl.). Chicago, London: University of Chicago Press.

Townsend, G. E. (1953). Short-term casework with clients under stress. Social Casework, 34(9), 392-398.

Traue, B. (2011). Coaching. Die Mobilisierung der Psyche 1775-1975. In S. Maasen, J. Elberfeld, P. Eitler M. Tändler (Hrsg.), Das beratene Selbst (S. 243-262). Bielefeld: transcript.

Walpuski, V.J. (2020a). Digitalisierte Beratung zur effizienteren Selbstoptimierung. Kritische Anmerkungen zu digitalen Formaten arbeitsbezogener Beratung aus einer Gouvernementalitätsperspektive. In R. Wegener, S. Ackermann, J. Amstutz, S. Deplazes, H. Künzli A. Ryter (Hrsg.), Coaching im Digitalen Wandel (S. 107-116). Vandenhoeck Ruprecht. https://doi.org/10.13109/ 9783666407420.107.

Walpuski, V.J. (2020b). Supervision als neues Element von Fachlichkeit in der Fürsorge nach 1945: Ein Beitrag zur historisch-kritischen Rekonstruktion der Einführung in Deutschland und Europa. In S. Businger M. Biebricher (Hrsg.), Von der paternalistischen Fürsorge zu Partizipation und Agency. Der gesellschaftli- 
che Wandel im Spiegel der Sozialen Arbeit und der Sozialpädagogik (S. 59-78). Zürich: Chronos.

Walpuski, V. J. (2021a). Cora Baltussen in den USA: Lernerfahrungen für die Supervision in den Niederlanden und Deutschland. Forum Supervision. Onlinezeitschrift Für Beratungswissenschaft und Supervision, 29(56), 76-97. https://doi.org/10.11576/fs-4067.

Walpuski, V. J. (2021b). Dora von Caemmerer: Von der Volkspflegerin zur Pionierin für Supervision. In C. Kuhlmann, R.-C. Amthor B. Bender-Junker (Hrsg.), Kontinuitäten und Diskontinuitäten Sozialer Arbeit nach dem Ende des Nationalsozialismus Bd. 1. Weinheim: Beltz Juventa.

Wandhoff, H. (2016). Was soll ich tun? Eine Geschichte der Beratung. Hamburg: Corlin.

Wastian, M., Poetschki, J. (2016). Zielklärung und Zielerreichung im Coaching. Coaching I Theorie Praxis, 2(1), 21-31. https://doi.org/ 10.1365/s40896-016-0011-3.
Weigand, W. (1990). Sozialarbeit - Supervision - Psychoanalyse: Ernst Federn, Wien, im Gespräch mit Wolfgang Weigand. Supervision, 8(18), 25-36.

Wieringa, C. F. (1970). Einzel- und Gruppensupervision im Rahmen der Sozialen Gruppenarbeit. In D. von Caemmerer (Hg.), Praxisberatung (Supervision). Ein Quellenband. Unter Mitarbeit von Marianne Fiedler, Renate Strömbach und Annedore Schultze (S. 290-314). Freiburg im Breisgau: Lambertus.

\section{Weiterführende Literatur}

Newstetter, W. I. (1951). Review: a comparison of diagnostic and functional casework concepts by Cora Kasius. The Annals of the American Academy of Political and Social Science, 27(3), 259-260. 